\title{
Testing for NRAS Mutations in Serous Borderline Ovarian Tumors and Low-Grade Serous Ovarian Carcinomas
}

\author{
Pawel Sadlecki $\mathbb{D}^{1},{ }^{1}$ Dariusz Grzanka, ${ }^{2}$ and Marek Grabiec ${ }^{1}$ \\ ${ }^{1}$ Department of Obstetrics and Gynecology, Collegium Medicum in Bydgoszcz, Nicolaus Copernicus University in Torun, \\ Bydgoszcz, Poland \\ ${ }^{2}$ Department of Clinical Pathomorphology, Collegium Medicum in Bydgoszcz, Nicolaus Copernicus University in Torun, \\ Bydgoszcz, Poland
}

Correspondence should be addressed to Pawel Sadlecki; pawelsadlecki@wp.pl

Received 18 October 2017; Revised 31 December 2017; Accepted 30 January 2018; Published 25 February 2018

Academic Editor: Dario de Biase

Copyright (C) 2018 Pawel Sadlecki et al. This is an open access article distributed under the Creative Commons Attribution License, which permits unrestricted use, distribution, and reproduction in any medium, provided the original work is properly cited.

The Idylla NRAS Mutation Test, performed on the Biocartis Idylla system, is an in vitro diagnostic tool for the qualitative assessment of 18 NRAS mutations in codons 12, 13,59, 61, 117, and 146. Low-grade serous ovarian cancer (LGSC) represents less than $10 \%$ of all serous ovarian carcinomas. LGSCs are believed to arise from preexisting cystadenomas or serous borderline tumors (SBOTs) that eventually progress to an invasive carcinoma. The molecular analysis of cancer-causing mutations and the development of targeted biological therapies constitute a milestone in the diagnosis and therapy of ovarian malignancies. According to some authors, NRAS may be an important oncogene for the progression of SBOT to a frankly invasive disease. The primary aim of this study was to verify if a fully integrated, real-time PCR-based Idylla system can be used for the rapid determination of the NRAS mutation status in patients with serous borderline ovarian tumors and low-grade serous ovarian carcinomas. The study included tissue specimens from 12 patients with histopathologically verified ovarian masses, operated on at the Department of Obstetrics and Gynecology, Nicolaus Copernicus University, Collegium Medicum in Bydgoszcz (Poland), between January 2009 and June 2012. The mean age of the study patients was 52.5 years (range 27-80 years). NRAS mutation in codon 13 (G13D, p.Gly13Asp; nucleotide: c.38G>A) was found in one patient, a woman with low-grade serous ovarian carcinoma. To the best of our knowledge, our experiment was the first published study using the novel Idylla NRAS Mutation Test for the evaluation of ovarian tumors in a clinical setting. The Idylla platform is an interesting ancillary first-line rapid and fully automated instrument to detect NRAS mutations in SBOTs and LGSCs. However, the clinical usefulness of this method still needs to be verified in larger groups of cancer patients.

\section{Introduction}

Point mutations in cancer cells can be detected with many various methods. The most popular method for the molecular characterization of genetic variants is direct sequencing, which can detect all potential variations, among them, base substitutions, insertions, and deletions. However, direct sequencing has some limitations when applied to clinical samples. First of all, it is not sensitive enough (10-30\%) to detect specific point mutations [1]. The analytic sensitivity of this method can be improved by pyrosequencing, high- resolution melting analysis, and real-time or allele-specific PCR assays [2, 3]. Application of direct sequencing as a routine method for cytological diagnosis in a hospital setting requires investment in expensive equipment and implementation of complicated procedures. Another factor limiting the application of this method in everyday clinical practice is long analytical times. This stimulated the search for a simple, rapid, specific, and sensitive method to detect point mutations. Recently, some new molecular assays for the detection of NRAS, KRAS, BRAF, and EGFR mutations have become available. These fully automated molecular 
diagnostic systems are suitable for quantitative allele-specific RT-PCR-based analyses and have been approved by the European Community for in vitro diagnostic use (CE-IVD label). As the assays provide high detection rates in NRAS, $K R A S, B R A F$, and EGFR genotyping, they can be used as a standardized method, even at diagnostic centers without a state-of-the-art molecular infrastructure $[4,5]$. Further, the testing does not require the involvement of highly skilled personnel, and using this instrument, even pathologists from less experienced laboratories can easily integrate morphological findings with molecular data, being crucial for further diagnostic and therapeutic decisions.

Ovarian cancer is one of the most common and lethal female malignancies worldwide. Regardless of the histological subtype, the standard treatment for ovarian cancer includes staging/debulking surgery and individualized platinum-based adjuvant chemotherapy [6, 7]. Low-grade serous ovarian cancer (LGSC) represents less than $10 \%$ of all serous ovarian carcinomas and is less sensitive to conventional platinum-based chemotherapy than high-grade serous ovarian cancers (HGSCs) $[8,9]$. LGSCs are believed to arise from preexisting cystadenomas or serous borderline tumors (SBOTs) that eventually progress to an invasive carcinoma. While most SBOTs do not have a typical phenotype of invasive carcinoma, microinvasion is not uncommon. In some studies, up to $60 \%$ of LGSCs were associated with SBOTs [10-12]. In line with the dualistic model of ovarian carcinogenesis, type I tumors are formed on a borderline background, from a distinct noninvasive (borderline or proliferating) precursor. Although KRAS/BRAF and TP53/BRCA mutations are very common in low- and high-molecular grade tumors, respectively, they are not found in all ovarian malignancies; this implies that other, still nonidentified pathway-related events, such as NRAS mutations, may be involved. NRAS is an established oncogene involved in the pathogenesis of other cancer types, including leukemia and melanoma $[13,14]$. According to some authors, NRAS may be also an important oncogene for the progression of SBOT to a frankly invasive disease [15]. The molecular analysis of cancer-causing mutations and the development of targeted biological therapies constitute a milestone in the diagnosis and therapy of ovarian malignancies. A few years ago, the classification of ovarian tumors was based mainly on the type of their primary tissue, histopathological, and clinical characteristics. Recently, however, oncological diagnosis has been also expanded to molecular features of cancer cells. Typically, molecular testing is performed whenever the detection of a certain alteration may have an impact on diagnosis and/or prognosis or if a targeted biological therapy is available.

The primary aim of this study was to verify if a fully integrated, real-time PCR-based Idylla system can be used for the rapid determination of NRAS mutation status in patients with serous borderline ovarian tumors and low-grade serous ovarian carcinomas.

\section{Material and Methods}

The study included tissue specimens from 12 patients with histopathologically verified ovarian masses, operated on at the Department of Obstetrics and Gynecology, Nicolaus
Copernicus University, Collegium Medicum in Bydgoszcz (Poland), between January 2009 and June 2012. The mean age of the study patients was 52.5 years (range $27-80$ years). All patients underwent surgical resection adequate for the clinical stage of their malignancy and, whenever necessary, received adjuvant platinum-based chemotherapy in line with current Polish guidelines [16]. The clinical stage of the ovarian tumors was determined in line with the guidelines of the International Federation of Gynecology and Obstetrics Committee on Gynecologic Oncology [17]. Based on histopathological examination of surgical specimens, five lesions were classified as low-grade serous ovarian carcinomas and seven as serous borderline ovarian tumors [18].

Clinicopathological characteristics of the study subjects are summarized in Table 1.

2.1. NRAS Mutation Assessment. Molecular studies of formalin-fixed paraffin-embedded (FFPE) specimens were conducted at the Department of Clinical Pathology, Nicolaus Copernicus University, Collegium Medicum in Bydgoszcz. NRAS mutation assay is one of the available diagnostic tests (CE-IVD) that can be conducted using the Idylla system (Biocartis, Mechelen, Belgium). The Idylla platform is a fully automated instrument based on a real-time PCR and fluorophore-based detection system, which, depending on the type of the used test cartridge, is suitable for the identification of NRAS, BRAF, KRAS, and EGFR mutations. The Idylla NRAS Mutation Test is suitable for the qualitative detection of 18 NRAS mutations: 5 mutations in codon 12 (exon 2), including p.G12C (c.34G>T), p.G12S (c.34G>A), p.G12D (c.35G>A), p.G12A (c.35G>C), and p.G12V (c.35G>T); 3 mutations in codon 13 (exon 2), among them p.G13D (c.38G $>$ A), p.G13V (c.38G $>$ T), and p.G13R (c.37G>C); 1 mutation in codon 59 (exon 3), A59T (c.175G>A); 4 mutations in codon 61 (exon 3), namely, p.Q61K (c.181C>A), p.Q61L (c.182A>T), p.Q61R (c.182A $>\mathrm{G})$, and p.Q61H $(\mathrm{c} .183 \mathrm{~A}>\mathrm{C}) ; 1$ mutation in codon 117 (exon 4), p.K117N (c.351G>C;c.351G>T); and 2 mutations in codon 146 (exon 4), p.A146T (c.436G >A) and p.A146V (c.437C>T), as well as NRAS total and BRAF total (wild-type mutations treated as a sample processing control; data not shown by the system).

Each specimen was examined by two independent pathologists to choose the most representative fragment of tumor tissue, with a minimum cellularity for NRAS-at least $10 \%$. Then, each $10 \mu \mathrm{m}$ section was placed between wet filter papers, transferred to an Idylla Test cartridge, and loaded onto the Idylla system. During the test, isolated nucleic acids were separated to 5 PCR chambers via microfluidic channels inside the cartridge (Idylla NRAS; Biocartis). To provide appropriate real-time PCR amplification and detection, all reagents were used in a dry form. Allele-specific targets were identified with a fluorescence-based detection system. A set of parameters describing generated PCR curves, for example, $\Delta \mathrm{Cq}$ value (calculated as the difference between the quantification cycle value $(\mathrm{Cq})$ of the gene control signal and the $\mathrm{Cq}$ of the mutant signal), is determined by the Idylla software. The limit of detection (LOD) is defined as the lowest NRAS mutation copy number consistently detected in $\geq 95 \%$ of the 
TABLE 1: Clinicopathological characteristics of the study subjects.

\begin{tabular}{|c|c|c|c|c|c|c|c|}
\hline lp & Histological type & Age & Menopausal status & Bilateral & $\begin{array}{c}\text { Size } \\
\text { Left/right }(\mathrm{cm})\end{array}$ & Stage & Mutation NRAS \\
\hline 1 & Cystadenoma papillare serosum proliferans & 48 & Premenopausal & + & $13 / 10$ & IC & No \\
\hline 2 & Cystadenoma papillare superficiale proliferans & 27 & Premenopausal & - & 18 & IA & No \\
\hline 3 & Cystadenoma papillare serosum proliferans & 47 & Premenopausal & - & 9 & IA & No \\
\hline 4 & Cystadenoma papillare serosum proliferans & 43 & Premenopausal & + & $7 / 6$ & IB & No \\
\hline 5 & Cystadenoma papillare serosum proliferans & 35 & Premenopausal & - & 5 & IA & No \\
\hline 6 & Cystadenoma papillare serosum proliferans & 72 & Postmenopausal & - & 9 & IA & No \\
\hline 7 & Cystadenoma papillare serosum proliferans & 60 & Postmenopausal & + & $4 / 5$ & IB & No \\
\hline 8 & Cystadenocarcinoma papillare serosum & 59 & Postmenopausal & - & 8 & IA & No \\
\hline 9 & Cystadenocarcinoma serosum & 50 & Premenopausal & + & $6 / 9$ & IB & Yes \\
\hline 10 & Cystadenocarcinoma serosum & 66 & Postmenopausal & - & 23 & IA & No \\
\hline 11 & Cystadenocarcinoma papillare serosum & 80 & Postmenopausal & - & 12 & IA & No \\
\hline 12 & Cystadenocarcinoma serosum & 43 & Premenopausal & - & 7 & IC & No \\
\hline
\end{tabular}

cases (with 95\% confidence), at an allelic frequency of 5\% for most prevalent NRAS mutations. A sample is classified as mutation-positive if the parameters of the generated PCR curve are within the validated range. Otherwise, the sample is classified as mutation-negative. The results, calculated by the dedicated Idylla software, were available after a $120 \mathrm{~min}$ run time [19].

The protocol of the study was approved by the local Bioethics Committee of the Nicolaus Copernicus University, Collegium Medicum in Bydgoszcz (decision number $\mathrm{KB}$ $413 / 2016$ ), and written informed consent was sought from each patient or her next of kin.

Statistical analysis of the results was carried out with the PQStat ver 1.6.4.112 package. Relationships between the presence of NRAS mutation and selected clinical parameters were analyzed with the Fisher exact test. Survivals were compared with log-rank, Wilcoxon-Breslow-Gehan, and Tarone-Ware tests. The results were considered significant at $p<0.05$ and highly significant at $p<0.01$.

\section{Results}

NRAS mutation in codon 13 (G13D, p.Gly13Asp; nucleotide: c. $38 \mathrm{G}>\mathrm{A}$ ) was found in one patient $(8.3 \%)$, a woman with low-grade serous ovarian carcinoma. The $\mathrm{Cq}$ and $\Delta \mathrm{Cq}$ values were 40.58 and 14.10 , respectively. PCR curves with corresponding $\mathrm{Cq}$ values for the positive and negative results of the Idylla NRAS Mutation Test are shown in Figures 1 and 2 , respectively. Selected clinical characteristics of the woman with NRAS mutation and patients who tested negatively for this genetic anomaly are compared in Table 2 , and the results of the survival analysis with NRAS mutation status as a predictor are shown in Table 3.

\section{Discussion}

The three human RAS genes encode four highly related 188to 189-amino acid proteins, designated as HRAS, NRAS, and KRAS (KRAS4A and KRAS4B). Ras proteins, located on the inner surface of the cell membrane, represent GDP/GTP- regulated switches that convey extracellular signals and are crucial for the intracellular signaling pathways being involved in fundamental cellular processes, such as cell polarity, proliferation, differentiation, adhesion, migration, and apoptosis $[20,21]$. Mutations in the neuroblastoma $R A S$ viral oncogene homolog (NRAS) constitutively activate intracellular signaling through a variety of pathways-most notably, the RAS-RAF-MAPK and PI3K-AKT pathways [22]. Although NRAS, KRAS, and HRAS share some structural and functional similarities, the most frequent $R A S$ alterations observed in human malignancies are KRAS mutations [23]. The frequency of $R A S$ mutants may vary considerably depending on the cancer type, with an estimated incidence of approximately $20 \%$ [23]. While amino acid residues G12, G13, and Q61 are the main mutational "hotspots" in colorectal malignancies, also other codons, among them 59, 117, and 146 , may be affected $[14,24]$. In melanomas, the most commonly mutated isoform of RAS is typically found at codons 12 and 61 or, less often, at codon 13. Mutant NRAS (Q61) interferes with the GTPase activity of RAS, locking it in its active conformation. In turn, NRAS (G12) and NRAS (G13) mutations affect the Walker A-motif (p-loop) of the protein, decreasing its sensitivity to GTPase-accelerating proteins. Mutations in G12/13 and Q61 can all be classified as activating mutations, yet they affect the NRAS protein in a distinct way, favoring the formation of GTP-bound, active RAS proteins $[25,26]$. Testing for NRAS mutations is now a standard of care in KRAS wild-type colorectal tumors [27]. Nearly $50 \%$ of the examined samples will return a wild-type KRAS result, and all these specimens should be tested for NRAS mutations [28]. Another interesting issue is the occurrence of comutations in RAS-RAF-MAPK pathway-associated genes, which may reflect the molecular heterogeneity of advanced colorectal cancer. Vagaja et al. reported the case of an adenocarcinoma in the caecum, containing a KRAS/NRAS comutation; the same comutation was also present in the contiguous high-grade tubulovillous adenoma [29]. The early occurrence of comutations implies that KRAS and NRAS may control different cellular functions; consequently, these two genes may exert a synergistic effect, since KRAS is primarily 

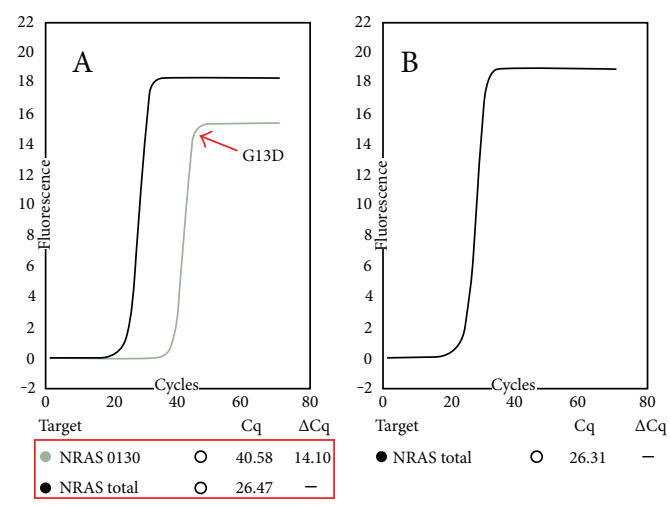

H\&E

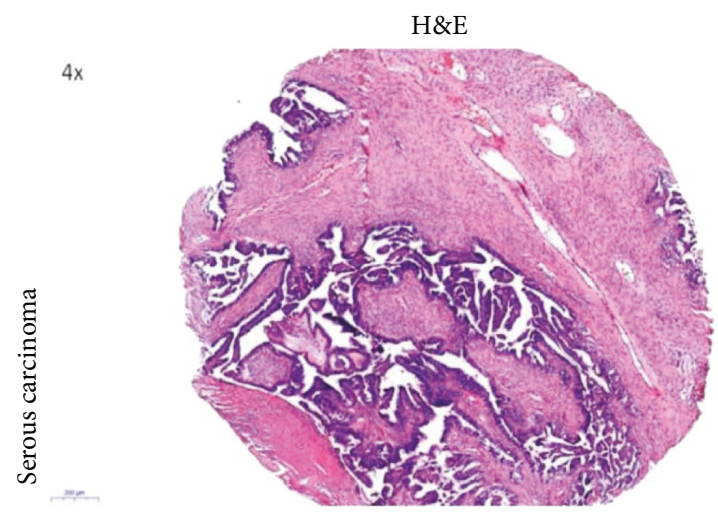

(b)

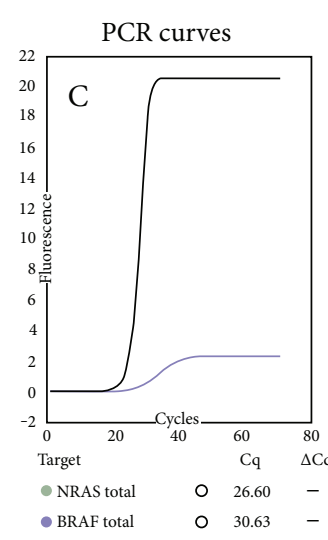

(a)
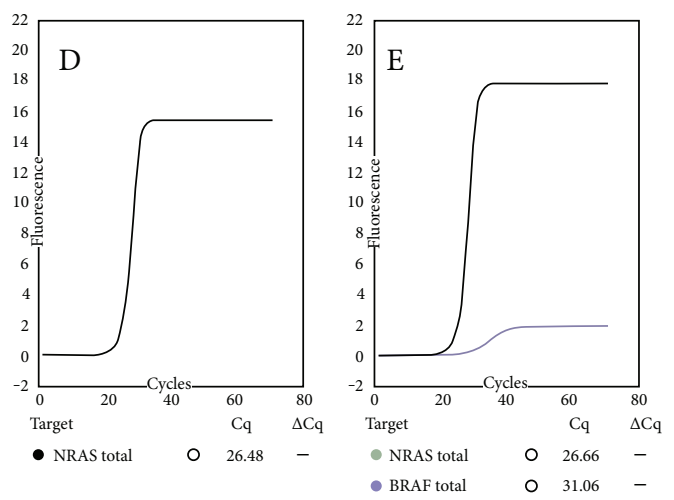

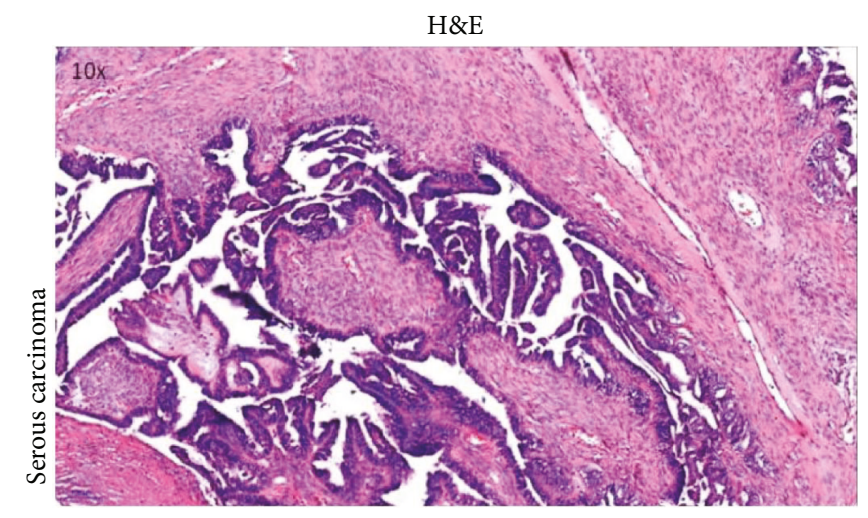

(c)

FIgURE 1: PCR curves from five separated PCR chambers (A-E) for the serous ovarian carcinoma which tested positively for NRAS mutation on the Idylla platform (a). Histological specimens from the same tumor, H\&E: $\times 4$ (b) and $\times 10$ (c).

involved in proliferation, whereas NRAS is known to regulate cell survival [30].

Our previous study demonstrated that although KRAS and $B R A F$ mutations never coexisted within the same ovarian tumor, a defect in one of those genes was present in 6 out of 7 SBOTs examined with the Idylla Mutation Test [31]. Ovarian malignancies acquire KRAS and BRAF mutations at very early stages of their progression, not infrequently before the SBOT stage, and additional driving events, such as the occurrence of NRAS mutations, have been postulated to facilitate the progression [15]. Xing et al. analyzed the NRAS mutation status of SBOTs, noninvasive LGSCs, and invasive LGSCs; interestingly, they did not find a NRAS mutation in either SBOTs or noninvasive LGSCs. According to these authors, the low prevalence of NRAS mutations implies that this gene may play a limited role in the development of LGSC [32]. However, in the study conducted by Emmanuel et al., activating NRAS mutations were found in 5 out of 58 (9\%) invasive serous epithelial ovarian carcinomas coexisting with a serous borderline tumor and in none of the 53 isolated SBOTs. The results of the same study suggested that Ras pathway mutations found in serous ovarian tumors may not be equivalent in terms of their pathogenicity [15]. This hypothesis seems to be also supported by the results of our present study, in which NRAS mutation was found in only one LGSC (8.3\%) and in none of SBOTs. We also identified NRAS mutation as a significant predictor of worse survival, and although our sample was too small to formulate any ultimate conclusions in this matter, still this association needs to be reported. However, the small size of our study group and lack of statistically significant associations between NRAS mutation status and histological type of the tumor put the importance of this finding into question.

NRAS mutations are also found in melanoma, colorectal cancer, follicular thyroid cancer, and acute myeloid leukemia [33-36]. In melanomas, NRAS mutations occur at a fairly consistent rate of $15-20 \%$ [37]. In melanoma patients, the presence of NRAS mutation was identified as a predictor of poorer outcomes and turned out to be associated with worse median overall survival (OS) [38]. Moreover, NRAS may serve as a biological marker to identify melanoma patients who can benefit from targeted therapies [39]. In colorectal cancer, NRAS mutations are found less often, in up to $4 \%$ of the cases, and also correlate with less favorable clinical outcome, enforcing the use of special treatment strategies $[40,41]$. For example, mutations within EGFR-signaling axis molecules, such as KRAS, NRAS, BRAF, and PIK3CA, predict resistance to anti-EGFR therapies [42]. Moreover, NRAS mutations are postulated to promote the malignant behavior of cancer cells in advanced colorectal malignancies; the results of both in vitro and animal studies suggest that this process can be reverted by silencing mutated codons [43]. 

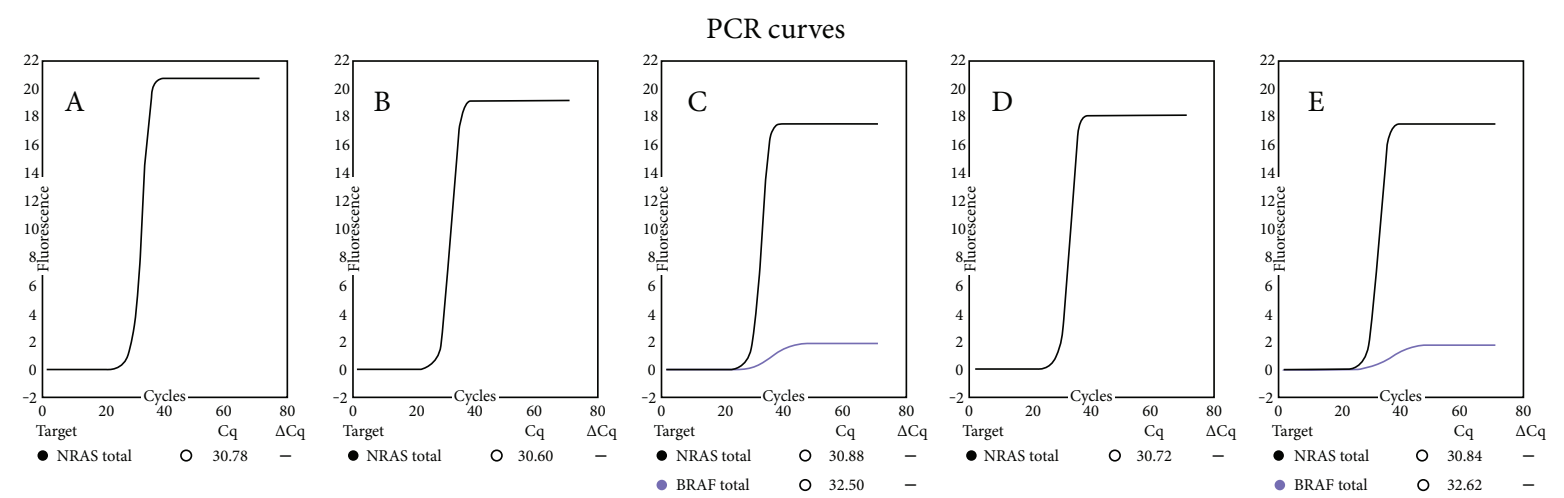

(a)

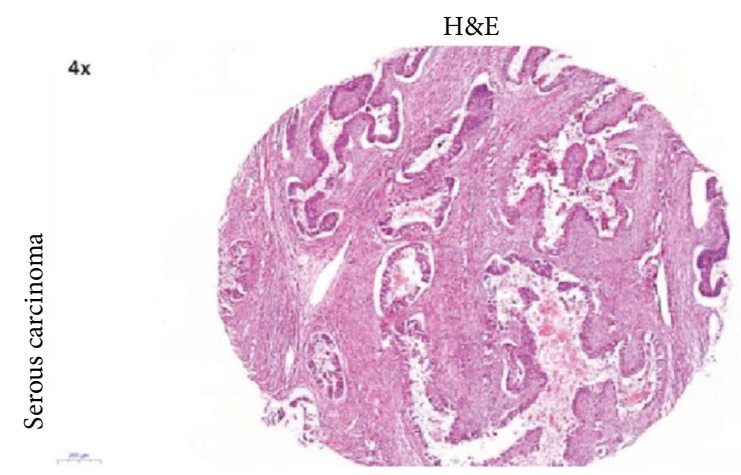

(b)

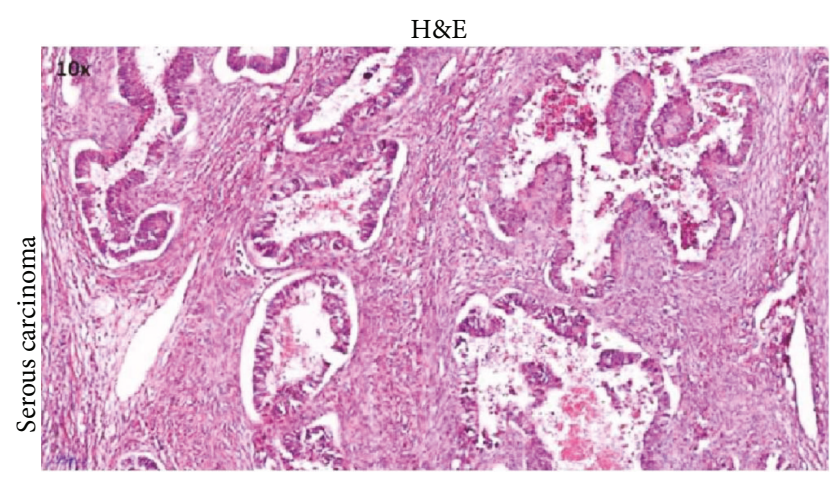

(c)

FIGURE 2: PCR curves from five separated PCR chambers (A-E) for a serous ovarian carcinoma which tested negatively for NRAS mutation on the Idylla platform (a). Histological specimens from the same tumor, H\&E: $\times 4$ (b) and $\times 10$ (c).

TABLE 2: Selected clinical characteristics of the study subjects, stratified according to NRAS mutation status.

(a)

\begin{tabular}{lccccc}
\hline \multirow{2}{*}{ NRAS mutation } & \multicolumn{2}{c}{ No } & \multicolumn{2}{c}{ Yes } & Fisher test \\
& $N$ & $\%$ & $N$ & $\%$ & \\
\hline Negative & 11 & $100 \%$ & 0 & $0 \%$ & \multirow{2}{*}{0.0833} \\
Positive & 0 & $0 \%$ & 1 & $100 \%$ & \\
\hline
\end{tabular}

(b)

\begin{tabular}{lccccc}
\hline \multirow{2}{*}{ NRAS mutation } & \multicolumn{4}{c}{ Bistological type } & \multirow{2}{*}{ Borderline } \\
& $N$ & $\%$ & $N$ & $\%$ & \\
\hline Negative & 7 & $100 \%$ & 4 & $80 \%$ & \multirow{2}{*}{0.4167} \\
Positive & 0 & $0 \%$ & 1 & $20 \%$ & \\
\hline
\end{tabular}

(c)

\begin{tabular}{|c|c|c|c|c|c|}
\hline \multirow{3}{*}{ NRAS mutation } & \multicolumn{4}{|c|}{ FIGO stage } & \multirow{3}{*}{ Fisher test } \\
\hline & \multicolumn{2}{|c|}{ IA } & \multicolumn{2}{|c|}{ Other } & \\
\hline & $N$ & $\%$ & $N$ & $\%$ & \\
\hline Negative & 7 & $100 \%$ & 4 & $80 \%$ & \multirow{2}{*}{0.4167} \\
\hline Positive & 0 & $0 \%$ & 1 & $20 \%$ & \\
\hline
\end{tabular}

No significant relationships were found between NRAS mutation status, mortality, histological type, and FIGO stage of the tumor.
TABLE 3: Results of survival analysis with NRAS mutation status as a predictor.

\begin{tabular}{lccc}
\hline Idylla NRAS & $\begin{array}{c}\text { Log-rank } \\
\text { test }\end{array}$ & $\begin{array}{c}\text { Wilcoxon-Breslow- } \\
\text { Gehan test }\end{array}$ & $\begin{array}{c}\text { Tarone- } \\
\text { Ware test }\end{array}$ \\
\hline $\begin{array}{l}\chi^{2} \text { statistic } \\
\begin{array}{l}\text { Degrees of } \\
\text { freedom }\end{array}\end{array}$ & 7.2735 & 6.8182 & 7.0455 \\
$p$-value & 1 & 1 & 1 \\
\hline
\end{tabular}

Irrespective of the type of statistical test, the presence of NRAS mutation was identified as a significant predictor of worse survival $(p<0.01)$.

These data on the effectiveness of targeted therapies in various malignancies harboring NRAS mutations justify further research on the role of these genetic anomalies in ovarian cancer [44].

The diagnostic platform Idylla seems to be an interesting alternative for the time-consuming and costly nextgeneration sequencing (NGS) techniques used for point mutation testing. The Idylla NRAS Mutation Test, performed on the Biocartis Idylla system, is an in vitro diagnostic tool for the qualitative assessment of 18 NRAS mutations in codons $12,13,59,61,117$, and 146 [19]. In the case of multiple mutations, only the one with the lowest $\Delta$ Cq value will be reported. Unlike other currently available technologies, the Idylla Mutation Test does not require manual preprocessing of the sample (deparaffinization, digestion of FFPE tissue, or 
DNA extraction), since all these procedures are integrated within a single-use cartridge [45]. Instead, whole FFPE tissue sections or macrodissected FFPE specimens are inserted into the cartridge and processed by the Idylla system; also, further stages, that is, real-time PCR-based mutation detection and result reporting, are fully integrated and automated [46]. Due to such characteristics of the platform, neither additional molecular infrastructure nor highly skilled personnel are required to perform the test. The accuracy of mutation assessment depends on several factors, such as tissue volume, DNA quality, and percentage of tumor cells, as well as on the specificity and sensitivity of a given test system [47]. According to some authors, the quality of the results obtained with the Idylla Mutation Test may be also influenced by the time elapsed between the sampling and the testing date. Weyn et al. observed a statistically significant association between the age of the samples that have been collected more than 4 years earlier and the likelihood of obtaining an invalid result with the Idylla KRAS Mutation Test [48]. Moreover, it should be remembered that currently available Idylla systems were designed to detect some specific mutations in NRAS, KRAS, $E G F R$, and $B R A F$ genes. Consequently, Idylla Mutation Tests are not capable of detecting each rare genetic defect; the presence of such rare mutations needs to be confirmed with ancillary molecular analyses, to prevent an inaccurate diagnosis and to implement the most effective treatment.

To the best of our knowledge, our experiment was the first published study using the novel Idylla NRAS Mutation Test for the evaluation of ovarian tumors in a clinical setting. The Idylla platform is an interesting ancillary first-line, rapid, and fully automated instrument to detect NRAS mutations in SBOTs and LGSCs. However, the clinical usefulness of this method still needs to be verified in larger groups of cancer patients.

\section{Conflicts of Interest}

The authors declare that they have no conflicts of interest.

\section{References}

[1] S. M. Anderson, "Laboratory methods for KRAS mutation analysis," Expert Review of Molecular Diagnostics, vol. 11, no. 6, pp. 635-642, 2011.

[2] J. C. Dudley, G. T. Gurda, L. H. Tseng et al., "Tumor cellularity as a quality assurance measure for accurate clinical detection of BRAF mutations in melanoma," Molecular Diagnosis \& Therapy, vol. 18, no. 4, pp. 409-418, 2014.

[3] C. T. Harbison, C. E. Horak, J. M. Ledeine et al., "Validation of companion diagnostic for detection of mutations in codons 12 and 13 of the KRAS gene in patients with metastatic colorectal cancer: analysis of the NCIC CTG CO.17 trial," Archives of Pathology \& Laboratory Medicine, vol. 137, no. 6, pp. 820-827, 2013.

[4] D. de Biase, C. de Luca, G. Gragnano et al., "Fully automated PCR detection of KRAS mutations on pancreatic endoscopic ultrasound fine-needle aspirates," Journal of Clinical Pathology, vol. 69, no. 11, pp. 986-991, 2016.

[5] P. Sadlecki, P. Walentowicz, M. Bodnar, A. Marszalek, M. Grabiec, and M. Walentowicz-Sadlecka, "Determination of BRAF V600E (VE1) protein expression and BRAF gene mutation status in codon 600 in borderline and low-grade ovarian cancers," Tumor Biology, vol. 39, no. 5, article $101042831770623,2017$.

[6] L. A. Torre, F. Bray, R. L. Siegel, J. Ferlay, J. Lortet-Tieulent, and A. Jemal, "Global cancer statistics, 2012," CA: A Cancer Journal for Clinicians, vol. 65, no. 2, pp. 87-108, 2015.

[7] J. S. Berek, C. Crum, and M. Friedlander, "Cancer of the ovary, fallopian tube, and peritoneum," International Journal of Gynecology \& Obstetrics, vol. 119, pp. S118-S129, 2012.

[8] J. P. Grabowski, P. Harter, F. Heitz et al., "Operability and chemotherapy responsiveness in advanced low-grade serous ovarian cancer. An analysis of the AGO Study Group metadatabase," Gynecologic Oncology, vol. 140, no. 3, pp. 457-462, 2016.

[9] C. Gourley, J. Farley, D. M. Provencher et al., "Gynecologic Cancer InterGroup (GCIG) consensus review for ovarian and primary peritoneal low-grade serous carcinomas," International Journal of Gynecological Cancer, vol. 24, no. 9, Supplement 3, pp. S9-S13, 2014.

[10] A. Malpica and M. T. Deavers, "Ovarian low-grade serous carcinoma involving the cervix mimicking a cervical primary," International Journal of Gynecological Pathology, vol. 30, no. 6, pp. 613-619, 2011.

[11] D. M. Gershenson, E. G. Silva, G. Tortolero-Luna, C. Levenback, M. Morris, and C. Tornos, "Serous borderline tumors of the ovary with noninvasive peritoneal implants," Cancer, vol. 83, no. 10, pp. 2157-2163, 1998.

[12] T. May, C. Virtanen, M. Sharma et al., "Low malignant potential tumors with micropapillary features are molecularly similar to low-grade serous carcinoma of the ovary," Gynecologic Oncology, vol. 117, no. 1, pp. 9-17, 2010.

[13] L. N. Kwong, J. C. Costello, H. Liu et al., "Oncogenic NRAS signaling differentially regulates survival and proliferation in melanoma," Nature Medicine, vol. 18, no. 10, pp. 1503-1510, 2012.

[14] I. A. Prior, P. D. Lewis, and C. Mattos, "A comprehensive survey of Ras mutations in cancer," Cancer Research, vol. 72, no. 10, pp. 2457-2467, 2012.

[15] C. Emmanuel, Y.-E. Chiew, J. George et al., "Genomic classification of serous ovarian cancer with adjacent borderline differentiates RAS pathway and TP53-mutant tumors and identifies NRAS as an oncogenic driver," Clinical Cancer Research, vol. 20, no. 24, pp. 6618-6630, 2014.

[16] A. Basta, M. Bidziński, A. Bieńkiewicz et al., "Rekomendacje Polskiego Towarzystwa Ginekologii Onkologicznej dotyczące diagnostyki i leczenia raka jajnika," 2015, http://ptgo.pl/dlalekarza/rekomendacje-i-zalecenia/.

[17] J. Prat and FIGO Committee on Gynecologic Oncology, "Staging classification for cancer of the ovary, fallopian tube, and peritoneum," International Journal of Gynaecology \& Obstetrics, vol. 124, no. 1, pp. 1-5, 2014.

[18] S. Hauptmann, K. Friedrich, R. Redline, and S. Avril, "Ovarian borderline tumors in the 2014 WHO classification: evolving concepts and diagnostic criteria," Virchows Archiv, vol. 470, no. 2, pp. 125-142, 2017.

[19] Technical sheet Idylla ${ }^{\mathrm{TM}}$ NRAS Mutation Test.

[20] P. P. Roux and J. Blenis, "ERK and p38 MAPK-activated protein kinases: a family of protein kinases with diverse biological functions," Microbiology and Molecular Biology Reviews, vol. 68, no. 2, pp. 320-344, 2004. 
[21] E. Castellano and J. Downward, "Role of RAS in the regulation of PI 3-kinase," Current Topics in Microbiology and Immunology, vol. 346, pp. 143-169, 2010.

[22] E. Hodis, I. R. Watson, G. V. Kryukov et al., "A landscape of driver mutations in melanoma," Cell, vol. 150, no. 2, pp. 251-263, 2012.

[23] M. Malumbres and M. Barbacid, " $R A S$ oncogenes: the first 30 years," Nature Reviews Cancer, vol. 3, no. 6, pp. 459-465, 2003.

[24] L. Haley, L. H. Tseng, G. Zheng et al., "Performance characteristics of next-generation sequencing in clinical mutation detection of colorectal cancers," Modern Pathology, vol. 28, no. 10, pp. 1390-1399, 2015.

[25] A. Daud and B. C. Bastian, "Beyond BRAF in melanoma," Current Topics in Microbiology and Immunology, vol. 355, pp. 99-117, 2012.

[26] I. V. Fedorenko, G. T. Gibney, and K. S. M. Smalley, "NRAS mutant melanoma: biological behavior and future strategies for therapeutic management," Oncogene, vol. 32, no. 25, pp. 3009-3018, 2013.

[27] E. Van Cutsem, A. Cervantes, B. Nordlinger, and D. Arnold, "Metastatic colorectal cancer: ESMO clinical practice guidelines for diagnosis, treatment and follow-up," Annals of Oncology, vol. 25, Supplement 3, pp. iiil-iii9, 2014.

[28] A. B. Benson 3rd, A. P. Venook, L. Cederquist, E. Chan, Y. J. Chen, and H. S. Cooper, "Colon Cancer, Version 1.2017, NCCN Clinical Practice Guidelines in Oncology," Journal of the National Comprehensive Cancer Network, vol. 15, no. 3, pp. 370-398, 2016.

[29] N. N. Vagaja, J. Parry, D. McCallum, M. A. Thomas, and J. M. Bentel, "Are all RAS mutations the same? Coexisting KRAS and NRAS mutations in a caecal adenocarcinoma and contiguous tubulovillous adenoma," Journal of Clinical Pathology, vol. 68, no. 8, pp. 657-660, 2015.

[30] K. M. Haigis, K. R. Kendall, Y. Wang et al., "Differential effects of oncogenic K-Ras and N-Ras on proliferation, differentiation and tumor progression in the colon," Nature Genetics, vol. 40, no. 5, pp. 600-608, 2008.

[31] P. Sadlecki, P. Antosik, D. Grzanka, M. Grabiec, and M. Walentowicz-Sadlecka, "KRAS mutation testing in borderline ovarian tumors and low-grade ovarian carcinomas with a rapid, fully integrated molecular diagnostic system," Tumor Biology, vol. 39, no. 10, article 101042831773398, 2017.

[32] D. Xing, Y. Suryo Rahmanto, F. Zeppernick et al., "Mutation of NRAS is a rare genetic event in ovarian low-grade serous carcinoma," Human Pathology, vol. 68, pp. 87-91, 2017.

[33] S. C. Chang, P. C. Lin, J. K. Lin et al., "Mutation spectra of common cancer-associated genes in different phenotypes of colorectal carcinoma without distant metastasis," Annals of Surgical Oncology, vol. 23, no. 3, pp. 849-855, 2016.

[34] N. Fakhruddin, M. Jabbour, M. Novy et al., "BRAF and NRAS mutations in papillary thyroid carcinoma and concordance in BRAF mutations between primary and corresponding lymph node metastases," Scientific Reports, vol. 7, no. 1, p. 4666, 2017.

[35] J. Yang, X. Lyu, X. Zhu et al., "Chromosome t(7;11)(p15;p15) translocation in acute myeloid leukemia coexisting with multilineage dyspoiesis and mutations in NRAS and WT1: a case report and literature review," Oncology Letters, vol. 13, no. 5, pp. 3066-3070, 2017.

[36] M. V. Heppt, T. Siepmann, J. Engel et al., "Prognostic significance of BRAF and NRAS mutations in melanoma: a German study from routine care," BMC Cancer, vol. 17, no. 1, p. 536, 2017.

[37] E. Muñoz-Couselo, E. Zamora Adelantado, C. Ortiz Vélez, J. Soberino-García, and J. M. Perez-Garcia, "NRAS-mutant melanoma: current challenges and future prospect," OncoTargets and Therapy, vol. 10, pp. 3941-3947, 2017.

[38] J. A. Jakob, R. L. Bassett Jr., C. S. Ng et al., "NRAS mutation status is an independent prognostic factor in metastatic melanoma," Cancer, vol. 118, no. 16, pp. 4014-4023, 2012.

[39] R. Dummer, D. Schadendorf, P. A. Ascierto et al., "Binimetinib versus dacarbazine in patients with advanced NRAS-mutant melanoma (NEMO): a multicentre, open-label, randomised, phase 3 trial," The Lancet Oncology, vol. 18, no. 4, pp. 435$445,2017$.

[40] Y. Wang, S. Velho, E. Vakiani et al., "Mutant N-RAS protects colorectal cancer cells from stress-induced apoptosis and contributes to cancer development and progression," Cancer Discovery, vol. 3, no. 3, pp. 294-307, 2013.

[41] J. Lasota, A. Kowalik, A. Felisiak-Golabek et al., "SP174, NRAS Q61R mutant-specific antibody, cross-reacts with KRAS Q61R mutant protein in colorectal carcinoma," Archives of Pathology \& Laboratory Medicine, vol. 141, no. 4, pp. 564-568, 2017.

[42] C. Therkildsen, T. K. Bergmann, T. Henrichsen-Schnack, S. Ladelund, and M. Nilbert, "The predictive value of KRAS, NRAS, BRAF, PIK3CA and PTEN for anti-EGFR treatment in metastatic colorectal cancer: a systematic review and meta-analysis," Acta Oncologica, vol. 53, no. 7, pp. 852-864, 2014.

[43] E. R. Fearon, "Molecular genetics of colorectal cancer," Annual Review of Pathology: Mechanisms of Disease, vol. 6, no. 1, pp. 479-507, 2011.

[44] P. A. Ascierto, D. Schadendorf, C. Berking et al., "MEK162 for patients with advanced melanoma harbouring NRAS or Val600 BRAF mutations: a non-randomised, open-label phase 2 study," The Lancet Oncology, vol. 14, no. 3, pp. 249-256, 2013.

[45] S. S. Sam, K. A. Lebel, C. L. Bissaillon, L. J. Tafe, G. J. Tsongalis, and J. A. Lefferts, "Automation of genomic DNA isolation from formalin-fixed, paraffin-embedded tissues," Pathology Research and Practice, vol. 208, no. 12, pp. 705-707, 2012.

[46] F. Janku, B. Claes, H. J. Huang et al., "BRAF mutation testing with a rapid, fully integrated molecular diagnostics system," Oncotarget, vol. 6, no. 29, pp. 26886-26894, 2015.

[47] J. R. Dijkstra, D. A. M. Heideman, G. A. Meijer et al., "KRAS mutation analysis on low percentage of colon cancer cells: the importance of quality assurance," Virchows Archiv, vol. 462, no. 1, pp. 39-46, 2013.

[48] C. Weyn, S. Van Raemdonck, R. Dendooven et al., "Clinical performance evaluation of a sensitive, rapid low-throughput test for KRAS mutation analysis using formalin-fixed, paraffin-embedded tissue samples," BMC Cancer, vol. 17, no. 1, p. 139, 2017. 


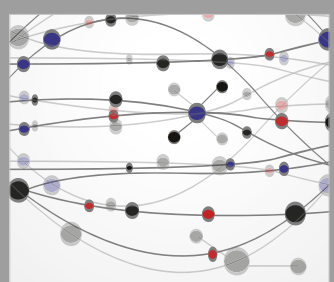

The Scientific World Journal
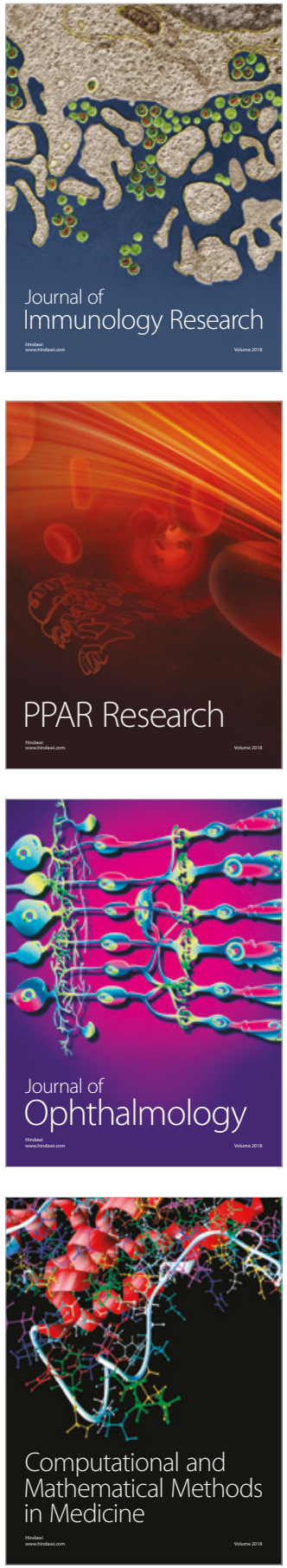

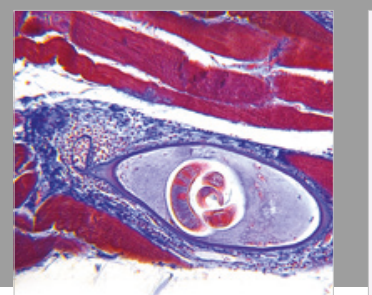

Gastroenterology Research and Practice

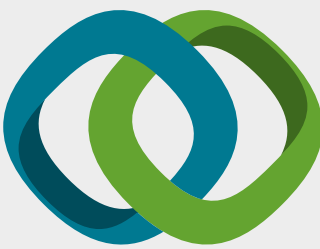

\section{Hindawi}

Submit your manuscripts at

www.hindawi.com
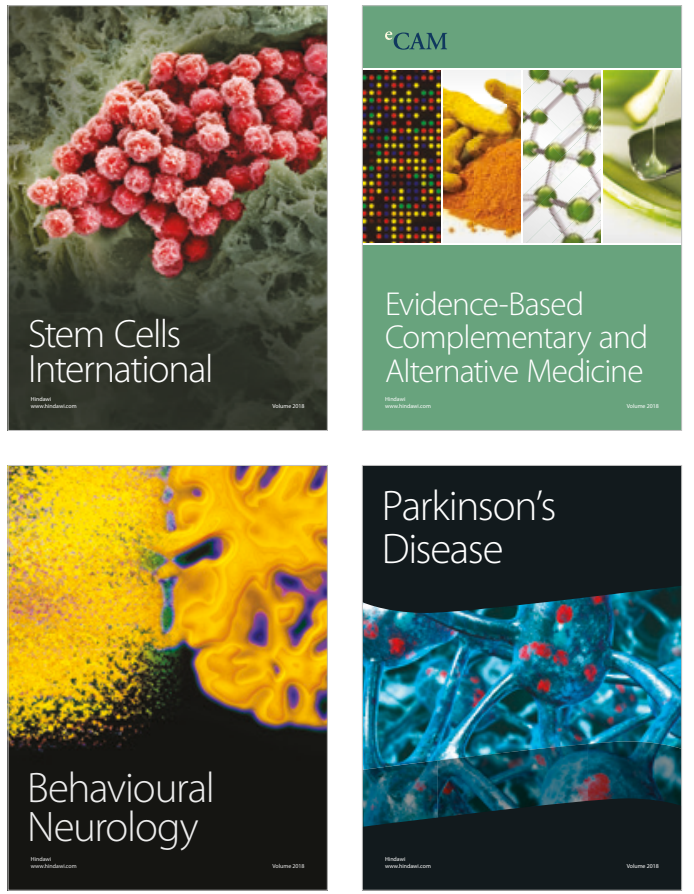

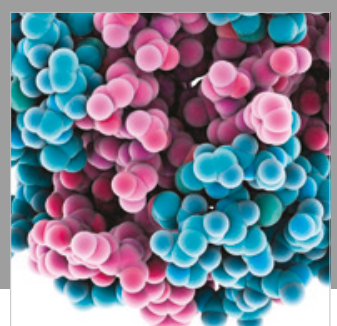

ournal of

Diabetes Research

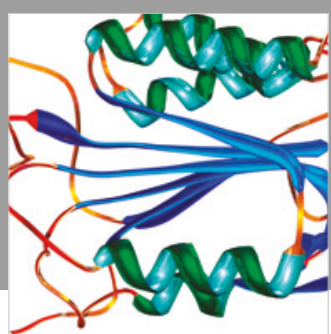

Disease Markers
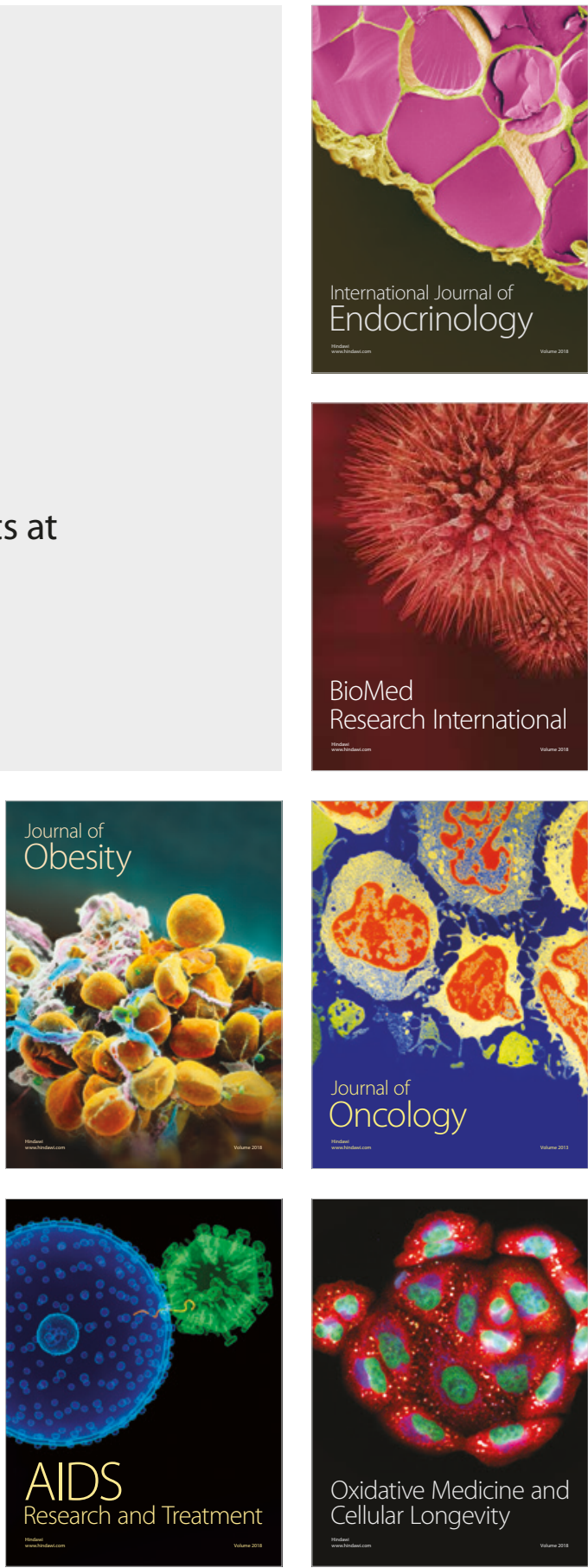